\title{
ACUTE MYOCARDIAL INFARCTION DUE TO HYPERSENSITYVITY TO ANTITUBERCULAR THERAPY
}

\author{
Mostafi $\mathrm{M}^{1}$, Hasan $\mathrm{ATMT}^{2}, \mathrm{Alim}_{\mathrm{MA}}^{3}$, Chakraborty B ${ }^{4}$
}

\begin{abstract}
Introduction
Vasculitis is a clinicopathological process characterized by blood vessel wall inflammation ${ }^{1}$. It can be primary or secondary to other systemic diseases ${ }^{2}$. The combination of tuberculosis and vasculitis was first described by Parish and Rhodes in $1967^{3}$. There are two general types of pulmonary tuberculosis-related vasculitis: leucocytoclastic vasculitis (a manifestation of pulmonary tuberculosis) and antitubercular medication-associated vasculitis (particularly with rifampicin therapy) ${ }^{4}$. Here a case is reported that presented with acute myocardial infarction due to coronary vasculitis secondary to antitubercular therapy.
\end{abstract}

\section{Case Report}

A 38-year-old male, known case of hypertension and dyslipidaemia being on regular medications, developed a remittent fever during early March, 2007. He was treated as a case of enteric fever with injection ceftriaxone and azithromycin for 7 days. There was no remission of fever and an antimalarial, a fixed-dose combination of artemether and lumefantrine, was added subsequently. There was no response. He had a few enlarged cervical lymph nodes, one of which was biopsied in Bumrungrad hospital, Bangkok and histopathologic examination revealed multiple caseous and noncaseous granulomas along with the presence of acid fast bacilli. Culture of the specimen yielded the growth of Mycobacterium tuberculosis sensitive to all 1st line antitubercular drugs. Under these circumstances, antitubercular therapy (ATT) with four drugs (rifampicin, isoniazid, pyrazinamide and ethambutol) were started. He returned to Bangladesh on the $17^{\text {th }}$ of March, 2007. He developed fever again on the $21^{\text {st }}$ of March. The body temperature had been rising everyday and it peaked to $105^{\circ} \mathrm{F}$ on the $26^{\text {th }}$ of March. The rise of temperature was accompanied by chills and rigors.

On the 6th day after the onset of fever, he experienced a period (3-4 hours) of hypotension and got himself admitted to Labaid Hospital, Dhaka. On the next day, he developed chest pain early in the morning and acute inferior myocardial infarction was diagnosed on the basis of characteristic ECG findings as well as a raised level of
troponin-I. Thrombolysis was done thereafter along with conventional treatment of myocardial infarction(MI) . His ATT was withheld.

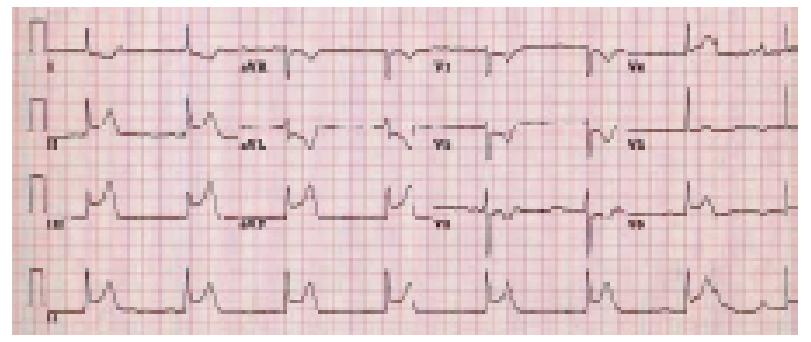

Figure 1: 12-lead ECG of the patient showing acute inferior MI.

In the hospital, he was not given any antibiotic and in spite of that he became afebrile after a period of two days. Following his recovery from the acute coronary event rifampicin and isoniazid was readministered on the 1st of April, 2007. Within an hour of taking the named drugs, he developed a severe allergic type of reaction featured by fever, chills, rigors, congested mucosae and skin rash. His blood pressure went up initially, but eventually it came down to $70 / 40 \mathrm{mmHg}$ and he required inotropic support. The antitubercular drugs were suspended again. The patient became alright again within the next two days. He was discharged with his usual cardiovascular drugs. Subsequently, his coronary angiogram (CAG) revealed insignificant single-vessel disease. He was prescribed medical management for his cardiac problem. Moreover, he was advised to undergo a streptomycin-based ATT excluding rifampicin. But he was too afraid to restart ATT. He consulted with a homeopathic physician and after 2 years of treatment he is now asymptomatic, there is no lymphadenopathy and there is also no laboratory evidence of disease activity.

\section{Discussion}

Acute myocardial infarction is the irreversible necrosis of heart muscle secondary to prolonged ischaemia. Although atherosclerosis is primarily responsible for most acute coronary syndrome cases, nonatherosclerotic causes of acute myocardial infarction should be considered in younger patients or if no evidence of

1. Col Mamun Mostafi MBBS, MACP, MRACP, FCPS, FRCP, Professor, Dept of Nephrology, CMH Dhaka, 2. Dr ATM Tanveer Hasan MBBS, Dept of Medicine, Dhaka Medical College 3. Brig Gen Md Abdul Alim MBBS, FACP, FCPS, Chief Physician, CMH Dhaka; 4. Prof Baren Chakraborty FCPS, FRCP, FACC, Consultanat Cadiologist, Labaid Cardiac Hospital, Dhaka 
atherosclerosis is noted. Such causes include coronary occlusion secondary to vasculitis, coronary emboli from sources, eg, infected cardiac valves, primary coronary vasospasm (vasospastic angina), cocaine use, or any other factor leading to mismatch of oxygen supply and demand $^{5}$.

The patient discussed here had four preexisting risk factors for developing an acute coronary syndrome, including male sex, hypertension, positive family history and dyslipidaemia. But it is interestingly noticeable that apart from acute myocardial infarction, the patient suffered from high fever, chills and rigors, congested mucosae and skin rash after the commencement of antitubercular therapy (ATT) and these quickly disappeared following cessation of antitubercular drugs. So all these observations suggest that there was some immunologically mediated event, more specifically a hypersensitivity reaction which was most likely to be responsible for the patient's post-ATT sickness.

In fact, several studies have described a relationship between the use of rifampicin (an essential component of ATT) and the onset of vasculitis ${ }^{6,7}$. Moreover, previous studies have suggested that anti-rifampicin antibodies contribute to the pathogenesis of vasculitis ${ }^{8}$. The pathophysiologic mechanism involved here is that antigen and antibody form large immune complexes and activate complement cascade. Moreover, leucocytes attracted to the site of reaction engulf the immune complexes and release pharmacologically active substances (including lysosomal enzymes) starting an inflammatory process. As a result small blood vessels are either damaged or blocked ${ }^{9}$.

A confounding factor with regard to this case is that vasculitis may also result from the deposition of immune complexes formed by antibodies against Mycobacterium tuberculosis proteins. The exixtence of such circulating immune complexes has been demonstrated in $56 \%$ of patients with active tuberculosis ${ }^{10}$. But, our patient developed the hypersensitivity features shortly after the commencement of ATT, rather than the very beginning of the 1st attack of pyrexia. So this excludes the possibility of direct leucocytoclastic vasculitis caused by Mycobacterium tuberculosis.

In fact, cutaneous leucocytoclastic vasculitis is a rare complication of $\mathrm{ATT}^{3}$. Coronary vasculitis secondary to ATT is even rarer and to the best of our knowledge, no such case has yet been reported.

\section{Conclusion}

Indeed, this case represents one of the multiple clinical outcomes of allergic drug reactions. It also highlights the importance of careful evaluation in order to elucidate the cause of acute myocardial infarction in young patients.

\section{References}

1. Sneller MC, Langford CA, Fauci AS. The Vasculitis Syndromes. In: Kasper DL, Braunwald E, Fauci AS, Hauser SL, Longo DL, Jameson JL, editors. Harrison's Principles of Internal Medicine. 16th ed. New York: McGraw-Hill;2005.p.2002-14.

2. Jennette JC, Falk RJ. Small-vessel vasculitis. N Engl J Med. 1997;337(21):1512-23.

3. Parish WE. Rhodes EL. Bacterial antigens and aggregated gamma globulin in the lesions of nodular vasculitis. Br J Dermatol. 1967; 79(3): 131-47.

4. Carvalho M, Dominoni RL, Senchechen D, Fernandes AF, Burigo IP, Doubrawa E. Cutaneous leukocytoclastic vasculitis accompanied by pulmonary tuberculosis. J Bras Pneumol 2008;34:745-8.

5. Grass S, Zafari AM. Myocardial Infarction. eMedicine Cardiology, http://www.emedicine.medscape.com. article 155919, Jul 20, 2010.

6. Chan CH, Chong YW, Sun AJ, Hoheisel GB. Cutaneous vasculitis associated with tuberculosis and its treatment. Tubercle 1990;71: 297300 .

7. Iredale JP, Sankaran R, Wathen CG. Cutaneous vasculitis associated with rifampin therapy. Chest 1989;96:215-6.

8. Poole G, Stradling P, Worlledge S. Potentially serious side effects of high-dose twice-weekly rifampicin. Br Med J 1971;3:343-7.

9. Bennet PN, Brown MJ. Unwanted effects and adverse drug reactions. Laurence Clinical Pharmacology. 9th ed. Churchill Livingstone: 2003. p.142.

10. Johnson NM, McNicol MW, Burton-Kee EJ, Mowbray JF. Circulating immune complexes in tuberculosis. Thorax 1981;36:610-7. 